\title{
Nursing diagnoses focused on universal self-care requisites
}

\author{
Carmen Queirós ${ }^{1,2,3,4}$ RN, MSC (D), Maria Antónia Taveira Cruz Paiva Silva ${ }^{2,5}$ RN, MSC, \\ PhD (D), Inês Cruz ${ }^{1,2,5}$ RN, MSC (D), Alexandrina Cardoso ${ }^{2,5}$ RN, MSC, PhD (i) \& \\ Ernesto J. Morais ${ }^{2,5}$ RN, MSC
}

I PhD Student, Invited Researcher, Rehabilitation Nurse, Institute of Biomedical Sciences Abel Salazar, University of Porto, Porto, 2 Associate Professor, CIDESI: ICN-Accredited Centre for Information Systems and ICNP ${ }^{\circledR}$ Research and Development, Nursing School of Porto, Porto, 3 PhD Student, Invited Researcher, Associate Professor, Health Sciences Research Unit: Nursing (UICISA: E), Coimbra Nursing School (ESEnfC), Coimbra, 4 Invited Researcher, Associate Professor, Department of Ortho-physiatry, Centro Hospitalar Universitário do Porto, Porto, $\mathbf{5}$ Invited Researcher, Associate Professor, Nursing School of Porto, Porto, Portugal

QUEIRÓS C., SILVA M.A.T.C.P., CRUZ I., CARDOSO A.\& MORAIS E.J. (2021) Nursing diagnoses focused on universal selfcare requisites. International Nursing Review 0, 1-13

Aims: (1) To identify and analyse diagnoses documented by nurses in Portugal within the scope of universal self-care requisites; (2) to determine the main problems with nursing diagnoses syntaxes for semantic interoperability purposes; and (3) to suggest unified nursing diagnoses syntaxes within the scope of universal self-care requisites.

Background/Introduction: Ageing societies and the increase in chronic diseases have led to significant concern regarding individuals' dependence to ensure self-care. ICNP is widely used by Portuguese nurses in electronic health records for documentation of nursing diagnoses and interventions.

Methods: A qualitative study using inductive content analysis and focus group: 1. nursing e-documentation content analysis and 2. focus group to explore implicit criteria or insights from content analysis results.

Results: From a corpus of analysis with 1793 nursing diagnoses, 432 nursing diagnoses centred on universal self-care requisites emerged from the content analysis. One hundred ten nursing diagnoses resulted from the application of new encoding criteria that emerged after a focus group meeting.

Conclusion: Results reveal that nursing diagnoses related to universal self-care requisites can emphasize the impairment or potentialities of the individuals performing self-care. It also shows a lack of consensus on nominating the nursing diagnoses of people with a deficit in universal self-care requisites, resulting in different diagnoses to express the same needs.

Implications for nursing practice: Representation of most relevant nursing diagnoses within the scope of universal self-care requisites. Implications for health policy: Incorporating standardized language into electronic health records is not enough for improving quality and continuity of care and semantic interoperability achievement. Electronic health records need to work with a nursing ontology in the backend to meet these requirements.

Keywords: Activities of Daily Living, Electronic Health Records, Focus Groups, Models, Nursing, Nursing, Nursing Diagnoses, Standardized Nursing Terminology

Correspondence address: Carmen Queirós, Avenida 1. ${ }^{\circ}$ de Dezembro, $1484^{\circ} \mathrm{Esq}$, 4590-505 Paços de Ferreira, Portugal; Tel: +351 914783048; Email:

carmenqueiros@gmail.com.

Conflict of interest: The authors declare that they have no known competing financial interests or personal relationships that could have appeared to influence the work reported in this paper.

Funding: This research received no specific grant from any funding agency in public, commercial or not-for-profit sectors.

\section{Introduction}

Ageing societies and the increase in chronic diseases have led to a significant concern on individuals' dependence to ensure self-care and their disabilities. In nursing theories (Meleis 2012), self-care is a core concept and a fundamental focus of clinical practice.

The theory of self-care deficit of Orem (1995) has self-care as a central concept, and was used as the theoretical and 
conceptual framework for our study. In this theory, self-care is a learned and intentional behaviour aimed to maintain the life, health and well-being of the individual. If performed effectively, it contributes to structural integrity, human functioning and development (Orem 1995). Individuals can make intentional choices and take care of themselves as self-care agents. Through a learning process, this lifelong ability may be conditional or non-existent. Under these circumstances, there is a need to involve others - family, friends or nurses. Orem (1995) defines conditions that guide the purposes of individuals to perform self-care - self-care requisites. There are three types of self-care requisites: development self-care requisites, health deviation self-care requisites and universal self-care requisites. This research focuses on universal self-care requisites. These are common to human beings during different life cycle stages and about vital processes and internal and external conditions that preserve functioning (Orem 1995). Commonly, universal requisites are called activities of daily living (Carpenito-Moyet 2011). Other authors conceptualize these as essential nursing care activities or fundamentals of care (Kitson et al. 2010; Kitson et al. 2013). In circumstances where self-care abilities are not adequate for self-care requisites, there is a self-care deficit, and nursing care is required (Orem 1995). Five help methods can be applied: (1) doing for, (2) orientation and guiding, (3) providing physical or psychological support, (4) providing and maintaining an environment for personal development and (5) teaching (Orem 1995).

All actions and interactions between nurses and individuals pursue active participation of the individual in self-care and the continuous satisfaction of self-care requisites. These are called nursing systems (Orem 1995), and there are three nursing systems: the wholly compensatory, the partially compensatory and the support education systems depending on the type of care individuals need. A deficit in universal self-care requisites leads to changes in everyday life such as bathing, dressing, going to the toilet and positioning (Brito 2016).

Nevertheless, there is a lack of evidence as to what nurses should do to provide optimal care and improve patient outcomes (Zwakhalen et al. 2018). Kalisch (2006) analysed a focus group of 107 nurses working in medical-surgical units of two hospitals and concluded that ambulation, turning, feedings and hygiene emerged from the nine regularly missed nursing care themes. A literature review by Jones et al. (2015), in which one of the aims was to describe the prevalence and patterns of unfinished care, corroborated that activities associated with mobility were among the top five nursing activities left unfinished. Moreover, this revealed the importance of self-care, particularly universal self-care requisites, as the core focus of nursing attention.
Patient care documentation is essential for patient safety and quality of care (Nursing \& Midwifery Council 2018). Nurses have been recording patient care since Florence Nightingale started to document soldiers' conditions during the Crimean War (Nightingale 1989). That information enabled her to identify the causes of infections as well as to prevent them and reduce mortality.

Technological development brought electronic health records (EHR), envisioning streamlined processes that improve the accuracy and efficiency of care and reduce human error (McCarthy et al. 2019). EHR is an electronic database whereby data are captured and can be analysed. This allows the right information on when, where, how and to whom it is needed to be provided 24/7 and improves decision-making, resulting in significantly better health and financial outcomes (Harrington 2019). EHRs can evolve into clinical decision support systems, giving health professionals information and guidance when a decision needs to be taken (Hardiker et al. 2019). An umbrella review conducted to reveal quality criteria, instruments and requisites for nursing documentation concluded that nurse documentation must align with the nursing process and use standard terminologies, user-friendly formats and systems, to achieve high quality (De Groot et al. 2019).

The nursing process reflects the clinical reasoning of nurses. It represents the construction of hypotheses based on data, information and knowledge (Goossen 2000). It encompasses assessing patients' needs, the definition of nursing diagnoses, prescription of nursing interventions, and descriptions and evaluation of results (Meleis 2012). A nursing diagnosis is a 'label given by a nurse to the decision about a phenomenon which constitutes the focus of nursing interventions' (International Council of Nurses 2016) and determines nursing care prescription. Incomprehensible diagnoses can negatively affect the quality of patient care and well-being (Paans et al. 2012).

Nurses can rely on terminologies to communicate with each other in a meaningful way. Terminology is defined as the language used by a discipline to describe its specific knowledge (NANDA-International 2015). It contains a set of predefined and agreed-upon terms structured by nurses (Tastan et al. 2014). Deciding upon which standards to use in EHR brings consistency to the process and accuracy, efficiency, reliability and comparability of health information at local, regional, national and international levels (Hardiker et al. 2019). To deliver excellent care quality and reduce healthcare costs across health systems in different countries, it is relevant that diverse computer systems share content and its meaning accurately and reliably - semantic interoperability (Gavrilov et al. 2019; Harrington 2019). This operation requires standardized terminologies and domain-driven 
ontologies. An ontology defines rules that govern the combination of terms and relationships. Domain experts develop these, and then users formulate queries with the specified concepts (Almeida \& Bax 2003). From this perspective, the development of a nursing ontology encompasses the use and organization of nursing terminology.

Portugal is at the forefront of eHealth in Europe (European Observatory on Health Systems \& Policies 2018). It stands out as a case study for the International Classification for Nursing Practice $^{\circledR}\left(\right.$ ICNP $\left.^{\circledR}\right)$ use in EHR (Paiva et al. 2014). One of the goals of the Nursing Practice Support System $\left(\mathrm{SAPE}^{\circledR}-\right.$ Sistema de Apoio à Prática de Enfermagem) was to advocate the continuous reflection of nurses' clinical practise (Silva 2006). Consequently, this software could encompass nursing care specificities by each ward and institution. After its development, the Nursing School of Porto made SAPE ${ }^{\circledR}$ available to the Portuguese Ministry of Health. In 2007, the Nursing Council (Ordem dos Enfermeiros) regulated the basic principles of EHR architecture and its functional technical requisites. One of the main aspects published was the mandatory use of ICNP ${ }^{\circledR}$ as a terminology reference of EHR (Ordem dos Enfermeiros 2007). This context has led to the widespread dissemination of ICNP ${ }^{\circledR}$ and EHR use by nurses in Portugal.

This study intended (1) to identify and analyse diagnoses documented by nurses in Portugal within the scope of universal self-care requisites; (2) to determine the main problems with nursing diagnoses syntaxes for semantic interoperability purposes; and (3) to suggest unified nursing diagnoses syntaxes within the scope of universal self-care requisites.

\section{Methods}

Despite the widespread use of NANDA and ICNP, terminologies are not enough for diverse computer systems to accurately and reliably share content and meaning. This operation also requires a nursing ontology. The extensive work we have done is the first step in developing detailed clinical models regarding universal self-care requisites. It is not our aim with this paper to relate our findings to established definitions, diagnoses and statements of NANDA, for example.

\section{Research design}

In January 2013, the utilization rate of EHRs that integrated ICNP ${ }^{\circledR}$ in Portuguese public hospitals was $93.1 \%$. Of these, $74.1 \%$ used SAPE $\circledast$, and $25.9 \%$ used other software developed by different companies, some of which incorporated ICNP ${ }^{\circledR}$ (73.3\%). In primary care, the utilization rate of information systems was very similar to those of public hospitals $-91 \%$ of the 348 existing Health Centers in Portugal used SAPE® at the time of analysis (Cardoso \& Sousa 2015).

Each institution that implemented SAPE $®$ created nursing diagnoses and interventions, and defined its relations using ICNP as a terminology reference. Although many different nursing diagnoses and interventions arose from this process, concerns about its reliability emerged and resulted in problematic semantic interoperability. The Portuguese Health Ministry, in pursuit of semantic interoperability, commissioned the Center for Information Systems Research and Development (CIDESI) at the Nursing School of Porto - an accredited centre for research of ICNP ${ }^{\circledR}$ by International Council of Nurses, an analysis of nursing documentation for all health institutions using SAPE® (Paiva et al. 2014). This study is a qualitative, exploratory and descriptive study developed in two phases: (1) nursing e-documentation content analysis (2) focus group to explore implicit criteria or insights undercover in content analysis results.

\section{Phase 1. Content analysis}

The content analysis method (Bardin 2018) comprised three steps: pre-analysis, exploration of material and treatment of results, inferences and interpretations. The Portuguese Health Ministry provided the Nursing School of Porto with all nursing diagnoses of each institution (hospitals and health centres) that used SAPE ${ }^{\circ}$ in 2013. Since the patient's self-care is relevant in all nursing settings, we did not exclude any setting when we analysed the nursing diagnoses.

The criteria used for selecting the material were as follows:

- Inclusion criteria: all nursing diagnoses with syntaxes which included 'self-care: hygiene' or 'self-care: bath' or 'washing' or 'self-care: personal arrangement' or 'self-care: clothing' or 'dressing or undressing' or 'eating' or 'self-care: drinking' or 'self-care: use of toilet' or 'self-care: physical activity' or 'sitting' or 'transferring' or 'positioning' or 'getting up' or 'walking with aid' or 'moving in a wheelchair';

- Exclusion criteria: all nursing diagnoses with syntaxes that fulfilled the inclusion criteria and incorporated in any order 'familiar caregiver' or 'family member role' or 'parent role' or 'ostomy'. An ongoing research project from CIDESI is studying the person living with a stoma (Silva et al. 2016); hence, we excluded 'ostomy'.

In the pre-analysis phase, the encoding rules were operationalized as follows:

- The terminology used was the ICNP ${ }^{\circledR}$ (International Council of Nurses 2013);

- Primitive concepts were used, mostly from the focus axis and not the pre-coordinated diagnostic concepts; 
- If obtained terms (focus or judgement or dimension) had the same term as the $\mathrm{ICNP}^{\circledR}$ (International Council of Nurses 2013), they were not changed, for example 'Self-Feeding';

- If obtained terms had a different expression, an update was made considering the term that represented a similar meaning at ICNP ${ }^{\circledR}$ (International Council of Nurses 2013), for example 'Moving in a Wheelchair', updated as to 'Wheelchair use';

- If obtained terms did not correspond to an ICNP® label (International Council of Nurses 2013), square brackets were used to identify that particular term, for example [adaptive strategies];

- The reference terminology model used for nursing diagnoses was ISO 18104 (International Organization for Standardization 2003). For instance, a nursing diagnosis is either a judgement on a focus or a judgement on the focus' dimension. A focus is an area of nursing attention. A judgement is an opinion regarding the focus and could be qualified as a degree, potentiality, acuity or timing. A dimension is a quality that provides perspective on focus. An entity to which a diagnosis refers to is a subject of the information. A site is a physical structure that specifies a focus' position (International Organization for Standardization 2003).

After a free reading of all extracted nursing diagnoses syntaxes, a new criterion was set resulting in the exclusion of:

- Syntaxes that reflected outcomes, for example 'Self-Feeding: Self-Feeding, not impaired';

- Misread diagnoses resulting from incomplete construction of nursing diagnosis, for example 'Pregnancy: selfcare' or 'Self-Turning: Skill Learning for the use of adaptive equipment for self-turning';

- The dissonance between focus and dimension or associated qualifier, for example 'Self-Care Drinking: Skill Learning of alternative communication, not Demonstrate';

- Diagnoses related to 'self-care: physical activity' and 'self-care: sleep-rest behaviour', to delimit the scope of analysis;

- Diagnoses focused on health deviation self-care requisites, such as 'Management of Therapeutic Regime: Dietary Knowledge, not Demonstrated';

- Diagnoses focused on developmental self-care requisites, for example 'Pregnancy Adaptation: knowledge on eating habits during pregnancy, not demonstrated';

- Diagnoses centred on factors related to a deficit in universal self-care requisites, for example 'Paresis: Skill Learning about Self-Turning'.
In the exploration stage, the corpus of analysis (Bardin 2018) was submitted to previously formulated rules. Each diagnosis was defined as a context unit, and a mixed procedure for encoding was adopted.

After categorizing all corpus, two external researchers, experts in content analysis with a $\mathrm{PhD}$ in nursing, reviewed our new categorization bringing reliability to the process.

\section{Phase 2. Focus group}

A focus group was conducted to explore implicit criteria or insights undercover in the results of the content analysis that could portray difficulties to semantic interoperability. The focus group followed Krueger \& Casey (2014) guidelines. A purposive sample of fifteen nurses was contacted by email. Criteria for inclusion were a) to be a nurse with a $\mathrm{PhD}$ or master's degree in nursing science and $b$ ) to have expertise in clinical reasoning and ICNP research - NursingOntos. The focus group meeting was held at the Nursing School of Porto. The facilitator was the primary researcher, a PhD student who worked as a registered nurse and had a master's degree in nursing science. The researcher was trained in qualitative research during the master's degree and $\mathrm{PhD}$ programme. The meeting began by identifying the study concept and its aims and consequently focus group meeting goals. The facilitator (CQ) used the following road map of questions: 'Is there any difference between each categorized term?' and 'Is it clinically relevant to use these concepts in diagnoses of universal self-care requisites?' and provoked a discussion among the participants. The meeting with $240 \mathrm{~min}$ was audiotaped with all participant's consent, and some notes were taken along the session. Results from the focus group were coded by two data coders. The inter-coder reliability was assured by using ICNP axis coding tree and ISO standard. Moreover, data saturation was obtained with a focus group meeting and no software was used for data analysis.

\section{Ethical considerations}

This study complied with the Declaration of Taipei (World Medical Association 2016) and the Declaration of Helsinki (World Medical Association 2013). Researchers had access to anonymized material, and participants of the focus group were informed about the objectives of the study before providing consent. All rights regarding data protection were respected. Transcripts kept individuals' anonymity. The audiotapes were only accessible to the first author and the transcripts to the authors. Ethical approval of the project was granted by the Ethics Committee of the Nursing School of Porto (no. 200/2020). 
Table 1 Corpus of analysis by universal self-care requisites focus

\begin{tabular}{lr}
\hline Focus axis & $\mathrm{n}$ \\
\hline & \\
Self-Hygiene & 252 \\
Self-Feeding & 207 \\
Self-toileting & 194 \\
Self-dressing or undressing & 177 \\
Self-transferring & 159 \\
Wandering & 124 \\
Self-turning & 118 \\
Walking using device & 98 \\
Drinking & 91 \\
Standing & 79 \\
Wheelchair use & 68 \\
Walking & 67 \\
Self-care & 41 \\
Self-Grooming & 40 \\
Self-bathing & 29 \\
Sitting & 25 \\
Self-washing & 15 \\
Knowledge & 1793 \\
Total & \\
\hline
\end{tabular}

\section{Results}

\section{Phase 1. Content analysis}

We retrieved 3398 nursing diagnoses syntaxes from SAPE®. A total of 1605 syntaxes (Table S1) were excluded. The remaining 1793 nursing diagnoses syntaxes composed our corpus of analysis (Table 1). Encoding rules (established previously) were applied, resulting in 432 nursing diagnoses syntax categories centred on universal self-care requisites (Table S2).

\section{Phase 2. Focus group and content analysis revisited}

In the focus group meeting, we exposed our results from content analysis and the diverse definitions from ICNP of the categorized primitive terms. We explored insights from the results of the content analysis focusing on the meaning of the nursing diagnoses and its syntax, specifically the ones that could reveal difficulties with semantic interoperability. The discussion centred on the differences between terms to express judgements and their degrees (Table S3) in the context of universal self-care requisites. Moreover, during the focus group, participants analysed the differences between the terms of ICNP that express dimensions and their specificities (Table S4). We provide samples from the comments made in the focus group, illustrating some of the findings, below are the exclusion and encoding criteria operationalized from this meeting:

- The exclusion of diagnoses focused on adaptation, 'are too abstract and comprehensive, have no clinical utility' P2, such as 'Self-Toileting: adaptation, impaired';

- Encoding all diagnoses which focus was wandering as walking. In Portuguese natural language, wandering is used as a synonym of walking. Even though the aimless roaming of wandering definition of ICNP did not capture the core definition of universal self-care requisites and patients' needs on these matters. In this case, the focus group participants decided that walking was the better term to use in universal self-care requisites context, for example 'Wandering impaired' should be encoded as 'Walking impaired';

- Encoding all diagnoses with a judgement of dependence or disability as impaired. Participants considered that 'dependence' illustrates the impact of the patient's self-care difficulties regarding its relationship with others. Furthermore, 'impaired' was considered more accurate to express patient problems, by themselves, on universal self-care requisites, for example 'Self-Feeding dependence' should be encoded as 'Self-Feeding impaired';

- Defining degrees of nursing diagnoses in a more accurate and less varying way since no data variability could sustain so many degrees of nursing diagnoses; hence, the degree should be less diverse. 'Mild or moderate degree is semantically similar with "partial", the same occurs with "high severe" or "severe" and "total" P4, for example 'Self-Feeding mild dependence' should be encoded as 'Self-Feeding partial impaired';

- Encoding all diagnoses with a judgement of low as [potential] for enhancing. In our raw material, we obtained diagnoses such as 'Moving in Wheelchair: skill learning for wheelchair use, not Demonstrated' and 'Moving in Wheelchair: ability (2.0) to use wheelchair, development potential'. Considering our encoding rules for phase 1 of content analysis, 'not demonstrated' was coded as low, and 'development potential' was coded as [potential] for enhancing. In the focus group meeting, the participants discussed these concepts and their definitions. They debated that these two different perspectives on judging the focus did not translate different nursing care needs. The emphasis considering the context units should be on the development of an individual's potentialities. Thus, the nursing diagnosis could guide nursing interventions. Considering Transitions Theory these diagnoses refers to a personal resource, hence readiness to improve knowledge/ability' P2;

- Encoding all diagnoses focused on learning capabilities or learning about adaptive strategies or techniques as 'ability'. 'It 
is legitimate that in these situations, nurses are referring to the instrumental ability of the individual, not to his learning process' P3, for example 'Self-Feeding: skill learning to [use adaptive strategies] for self-feeding, low' was encoded as 'SelfFeeding: [potential] for enhancing the ability for Self-Feeding';

- Encoding all diagnoses focused on cognitive learning or knowledge about a technique as 'ability'. Participants discussed the ambiguity in using these concepts - cognitive learning about a technique and knowledge about a technique. They stated that the field of information to be given regarding technique represents self-care performance by itself. Hence, in this context of universal self-care requisites, it should not be possible to have in the EHR different nursing diagnoses that could portray similar meanings with nurses prescribing similar interventions, for example 'Self-dressing or undressing: knowledge about technique, low' was encoded as 'Self-dressing or undressing: [potential] for enhancing ability for self-dressing or undressing';

- Encoding all diagnoses focused on knowledge about adaptive strategies as 'knowledge about device', for example 'Self-dressing or undressing: knowledge about [adaptive strategies], low' was encoded as 'Self-dressing or undressing: [potential] for enhancing knowledge about device';

- Encoding all diagnoses focused on willpower as 'self-efficacy'. The focus group participants discussed the term 'willpower' in the context of universal self-care requisites. They explored the idea that the absence of willpower is a sign of absent successful experiences resulting in data to another focus - self-efficacy.

The main problems found in nursing diagnoses syntaxes for semantic interoperability purposes were conceptual redundancy between categorial structures for representation of nursing diagnoses leading to ambiguity and different levels of granularity and comprehensiveness between nursing diagnoses. After the focus group meeting, we revisited our corpus and applied the new encoding criteria to it. This process conducted to 110 nursing diagnoses syntax categories (Table 2). In Figure 1, we represent the process we undertook with the summary of findings.

\section{Discussion}

The unified nursing diagnoses syntaxes resulting from this study support semantic interoperability in the documentation of individuals' needs on universal self-care requisites. With the focus group participants' contributions, we reduced variance, degrees and collapsed categories of the nursing diagnoses categories. In our results, we found concatenation of focus and different judgements, for example 'low' and 'impaired' portrayed similar meanings to describe the same phenomena. This resulted in different diagnoses being used by nurses to express the same needs. Semantic propagation and redundancy between terms adopted for focus and judgements explained most of the disparity found before and after the focus group content analysis. The core focus of nursing attention should be expected to have clear standard terms to describe patient needs. However, this is not the case. Kitson et al. (2010) also expressed that essential elements of patient care do not have precise metrics. Also, there is a lack of agreement about what descriptors to use in terminologies.

From another perspective, a locally customized EHR, even with the use of standardized terminology, will provide users with familiar terms and data that have always been collected regardless of its clinical utility (Harrington 2019). Neves \& Parente (2019), Goncalves et al. (2018) and Cruz et al. (2016) reported the same results in content analysis of Portuguese nursing diagnoses in the neuromuscular process, mental health records and on the management of treatment regimen, respectively. This suggests that ICNP nursing care planning customization should not happen at an institutional level. Keenan \& Wilkie (2014) achieved similar results with locally customized EHR using NANDA, NIC and NOC linkage.

Two perspectives of reasoning about the need on universal self-care requisites arose from the unified nursing diagnoses: (1) absence or deficit of self-care or (2) an opportunity of the individual to develop abilities, or knowledge to deal with this deficit. On the one hand, the ICNP ${ }^{\circledR}$ is not grounded in a specific nursing theory or model (Silva 2006). However, our diagnoses underly Orem (1995) and claim that all actions and interactions between nurses and individuals should pursue active participation of the individual in self-care, for example 'Wheelchair use: [potential] for enhancing ability for wheelchair use'. As well as continuous and effective satisfaction of self-care requisites, when self-care needs exceed individual capacities, for example 'Self-toileting: impaired'. Our findings highlight the individual need to acquire knowledge and master skills for managing a new challenging condition, for example 'Self-transferring: [potential] for enhancing knowledge about device', 'Self-turning: [potential] for enhancing ability for self-turning' and 'Self-toileting: [potential] for enhancing ability for self-toileting using device'. Hence, guiding the implementation of nursing interventions related to the 'orientation and guidance' and 'teaching' methods pointed by Orem (1995). The possibility of making different nursing diagnoses focusing on patient potential regarding self-care abilities allows nurses to distinguish different needs, such as the ones of individuals with an impairment in self-care and the one of those who can improve this deficit. A nursing diagnosis determines nursing care interventions. Many of 
Table 2 Final diagnoses categories (+ICNP codes)

Final diagnoses categories (+ICNP codes)

Self-Feeding: neglect $(10017730+10033462)$

Self-Feeding: partial impaired $(10017730+10014081+10012938)$

Self-Feeding: [potential] for enhancing self-efficacy for self-feeding $(10017730+[$ potential] $+10006945+10024911+10017730)$

Self-Feeding: [potential] for enhancing ability for self-feeding $(10017730+$ [potential] $+10006945+10000034+10017730)$

Self-Feeding: [potential] for enhancing ability for self-feeding using device $(10017730+[$ potential $+10006945+10000034+10017730+10005869)$

Self-Feeding: [potential] for enhancing knowledge about device $(10017730+[$ potential] $+10006945+10011042+10005869)$

Self-Feeding: [potential] for enhancing knowledge about food preparation $(10017730+$ [potential] $+10006945+10011042+10008117)$

Self-Feeding: total impaired $(10017730+10019876+10012938)$

Self-Feeding: impaired $(10017730+10012938)$

Self-Feeding: interrupted $(10017730+10010519)$

Walking Using Device: partial impaired $(10020903+10014081+10012938)$

Walking Using Device: [potential] for enhancing self-efficacy for walking using device $(10020903+$ [potential] $+10006945+10024911+10020903)$

Walking Using Device: [potential] for enhancing ability for walking using device $(10020903+$ [potential] $+10006945+10000034+10020903)$

Walking Using Device: [potential] for enhancing knowledge about device $(10020903+$ [potential] $+10006945+10011042+10005869)$

Walking Using Device: (total) impaired $(10020903+10019876+10012938))$

Walking Using Device: impaired (10020903 + 10012938)

Walking: (partial) impaired $(10020886+10014081+10012938)$

Walking: [potential] for enhancing self-efficacy for walking $(10020886+[$ potential] $+10006945+10024911+10020886)$

Walking: [potential] for enhancing ability for walking $(10020886+$ [potential] $+10006945+10000034+10020886)$

Walking: total impaired $(10020886+10019876+10012938)$

Walking: impaired $(10020886+10012938)$

Self-Grooming: neglected $(10017753+10033462)$

Self-Grooming: partial impaired $(10017753+10014081+10012938)$

Self-Grooming: [potential] for enhancing ability for self-grooming $(10017753+[$ potential] $+10006945+10000034+10017753)$

Self-Grooming: [potential] for enhancing ability for self-grooming using device $(10017753+$ potential] $+10006945+10000034+10017753+10005869)$

Self-Grooming: [potential] for enhancing knowledge about device $(10017753+[$ potential] $+10006945+10011042+10005869)$

Self-Grooming: total impaired $(10017753+10019876+10012938)$

Self-Grooming: impaired $(10017753+10012938)$

Self-care: neglected $(10017661+10033462)$

Self-care: partial impaired $(10017661+10014081+10012938)$

Self-care: [potential] for enhancing self-efficacy $(1001766+[$ potential] $+10006945+10024911)$

Self-care: [potential] for enhancing ability to perform self-care $(10017661+$ [potential] $+10006945+10000034+10023729$

Self-care: [potential] for enhancing awareness about [need for change] $(10017661+$ [potential] $+10006945+10003083$

Self-care: total impaired $(10017661+10019876+10012938)$

Self-care: impaired $(10017661+10012938)$

Drinking: partial impaired $(10006276+10014081+10012938)$

Drinking: [potential] for enhancing ability for drinking $(10006276+$ potential] $+10006945+10000034+10006276)$

Drinking: [potential] for enhancing ability for drinking using device $(10006276+[$ potential] $+10006945+10000034+10006276+10005869)$

Drinking: [potential] for enhancing knowledge about device $(10006276+$ [potential] $+10006945+10011042+10005869)$

Drinking: total impaired $(10006276+10019876+10012938)$

Drinking: impaired $(10006276+10012938)$

Self-Hygiene: partial impaired $(10017769+10014081+10012938)$

Self-Hygiene: neglected $(10017769+10033462)$

Self-Hygiene: [potential] for enhancing self-efficacy for self-hygiene $(10017769+$ [potential] $+10006945+10024911+10017769)$

Self-Hygiene: [potential] for enhancing ability for self-hygiene $(10017769+$ [potential] $+10006945+10000034+10017769)$

Self-Hygiene: [potential] for enhancing ability for self-hygiene using device $(10017769+$ [potential] $+10006945+10000034+10017769+10005869)$

Self-Hygiene: [potential] for enhancing ability to perform oral care $(10017769+[$ potential] $+10006945+10000075+10032184)$

Self-Hygiene: [potential] for enhancing knowledge about device $(10017769+[$ potential] $+10006945+10011042+10005869)$

Self-Hygiene: impaired $(10017769+10012938)$

Self-Hygiene: interrupted $(10017769+10010519)$ 
Table 2 Continued

Final diagnoses categories ( + ICNP codes)

Self-Hygiene: total impaired $(10017769+10019876+10012938)$

Standing: partial impaired $(10018755+10014081+10012938))$

Standing: [potential] for enhancing self-efficacy for standing $(10018755+$ [potential] $+10006945+10024911+10018755)$

Standing: [potential] for enhancing ability for standing $(10018755+$ [potential] $+10006945+10000034+10018755)$

Standing: [potential] for enhancing ability for standing using device $(10018755+[$ potential] $+10006945+10000034+10018755+10005869)$

Standing: [potential] for enhancing knowledge about device $(10018755+$ [potential] $+10006945+10011042+10005869)$

Standing: total impaired $(10018755+10019876+10012938)$

Standing: impaired $(10018755+10012938)$

Self-washing: partial impaired $(10017846+10014081+10012938)$

Self-washing: total impaired $(10017846+10019876+10012938)$

Sitting: partial impaired $(10018195+10014081+10012938)$

Sitting: [potential] for enhancing ability for sitting $(10018195+[$ potential] $+10006945+10000034+10018195)$

Sitting: [potential] for enhancing ability for sitting using device $(10018195+[$ potential] $+10006945+10000034+10018195+10005869)$

Sitting: [potential] for enhancing knowledge about device $(10018195+$ [potential] $+10006945+10011042+10005869)$

Sitting: total impaired $(10018195+10019876+10012938)$

Sitting: impaired $(10018195+10012938)$

Self-bathing: partial impaired $(10017657+10014081+10012938)$

Self-bathing: [potential] for enhancing ability for self-bathing $(10017657+[$ potential] $+10006945+10000034+10017657)$

Self-bathing: [potential] for enhancing ability for self-bathing using device $(10017657+$ [potential] $+10006945+10000034+10017657+10005869)$

Self-bathing: [potential] for enhancing knowledge about device $(10017657+[$ potential] $+10006945+10011042+10005869)$

Self-bathing: total impaired $(10017657+10019876+10012938)$

Self-bathing: impaired $(10017657+10012938)$

Self-transferring: partial impaired $(10017822+10014081+10012938)$

Self-transferring: [potential] for enhancing ability for self-transferring $(10017822+$ [potential] $+10006945+10000034+10017822)$

Self-transferring: [potential] for enhancing ability for self-transferring using device

$(10017822++[$ potential $]+10006945+10000034+10017822+10005869)$

Self-transferring: [potential] for enhancing knowledge about device $(10017822+$ [potential] $+10006945+10011042+10005869)$

Self-transferring: total impaired $(10017822+10019876+10012938)$

Self-transferring: impaired $(10017822+10012938)$

Wheelchair use: partial impaired $(10021075+10014081+10012938)$

Wheelchair use: [potential] for enhancing self-efficacy for wheelchair use $(10021075+$ [potential] $+10006945+10024911+10021075)$

Wheelchair use: [potential] for enhancing ability for wheelchair use $(10021075+[$ potential] $+10006945+10000034+10021075)$

Wheelchair use: [potential] for enhancing knowledge about device $(10021075+$ [potential] $+10006945+10011042+10005869)$

Wheelchair use: total impaired $(10021075+10019876+10012938)$

Wheelchair use: impaired $(10021075+10012938)$

Self-toileting: partial impaired $(10017814+10014081+10012938)$

Self-toileting: [potential] for enhancing self-efficacy for self-toileting $(10017814+$ [potential] $+10006945+10024911+10017814)$

Self-toileting: [potential] for enhancing ability for self-toileting $(10017814+[$ potential] $+10006945+10000034+10017814)$

Self-toileting: [potential] for enhancing ability for self-toileting using device $(10017814+$ [potential] $+10006945+10000034+10017814+10005869)$

Self-toileting: [potential] for enhancing knowledge about device $(10017814+[$ potential] $+10006945+10011042+10005869)$

Self-toileting: total impaired $(10017814+10019876+10012938)$

Self-toileting: impaired $(10017814+10012938)$

Self-toileting: interrupted $(10017814+10010519)$

Self-dressing or undressing: neglected $(10017748+10033462)$

Self-dressing or undressing: partial impaired $(10017748+10014081+10012938)$

Self-dressing or undressing: [potential] for enhancing self-efficacy for self-dressing or undressing

$(10017748+[$ potential $]+10006945+10024911+10017748)$

Self-dressing or undressing: [potential] for enhancing ability for self-dressing or undressing

$(10017748+[$ potential $]+10006945+10000034+10017748)$

Self-dressing or undressing: [potential] for enhancing ability for self-dressing or undressing using device

$(10017748+[$ potential $]+10006945+10000034+10017748+10005869)$ 
Table 2 Continued

Final diagnoses categories ( + ICNP codes)

Self-dressing or undressing: [potential] for enhancing knowledge about device $(10017748+$ [potential] $+10006945+10011042+10005869)$

Self-dressing or undressing: [potential] for enhancing knowledge for regulating clothing

$(10017748+[$ potential $]+10006945+10011042+10016613+10002589)$

Self-dressing or undressing: total impaired $(10017748+10019876+10012938)$

Self-dressing or undressing: impaired $(10017748+10012938)$

Self-dressing or undressing: interrupted $(10017748+10010519)$

Self-turning: partial impaired $(10017833+10014081+10012938)$

Self-turning: [potential] for enhancing self-efficacy for self-turning $(10017833+[$ potential] $+10006945+1002491+10017833)$

Self-turning: [potential] for enhancing ability for self-turning $(10017833+$ [potential] $+10006945+10000034+10017833)$

Self-turning: [potential] for enhancing ability for self-turning using device $(10017833+$ [potential] $+10006945+10000034+10017833+10005869)$

Self-turning: [potential] for enhancing knowledge about device $(10017833+$ [potential] $+10006945+10011042+10005869)$

Self-turning: total impaired $(10017833+10019876+10012938)$

Self-turning: impaired $(10017833+10012938)$

Self-turning: interrupted $(10017833+10010519)$

interventions implemented towards universal self-care requisites needs' aim to improve or to prevent further impairment (Friedman et al. 2014). In our findings, the nursing diagnoses point out to the individual's resources and its potentiality to overcome the impairment.

The trigger for transitioning from dependency to an independence situation starts when the individual becomes aware of the changes that occurred, such as the confrontation with the necessary self-care activities that he could not perform (Brito 2016). Our results undertake this idea, for example 'Self-care: [potential] for enhancing awareness about [need for change]'. Chick \& Meleis (1986) claim that individuals must be aware of what changes occur to initiate a transition experience. Transition theory is a middle-range theory that describes the types and patterns of transitions and their properties, such as awareness. On transition theory is also described the conditions that could facilitate or inhibit transitions - such as knowledge, and patterns of response, outcome indicators and nursing therapeutics (Meleis 2012).

Transition theory (Meleis 2012) contextualizes along with self-care deficit theory, our results. The nursing diagnoses obtained in our study reflect a collection of skills and knowledge that individuals need to manage when universal self-care requisites are in deficit, which is also supported by Kitson et al. (2010). We recognize clinical conceptual models absorbed into Portuguese clinical practices and its knowledge domain and content in EHR. This could reveal the specificity of nursing care to policymakers and health gains produced by nurses (Spigolon \& Moro 2012) if the terms used to describe nursing diagnoses were accurate and reliable (Tastan et al. 2014).
Nursing diagnostic accuracy relies upon nurses' competence in critical thinking and reasoning skills. We believe that through unified nursing diagnoses syntaxes, we balance granularity and the comprehensiveness of their descriptions. This paper did not aim to describe the essential date to define each nursing diagnoses and its degrees. However, we believe that a detailed data structure should enlighten these (Paans et al. 2012) and should be integrated into EHR. Sousa et al. (2012) claimed that detailed electronic data records grounded in terminologies could assist nurses' decision-making in improving patient care and safety. This will prevent the use of the different nursing diagnoses to express the same needs and its opposite situation. Nonetheless, research is needed to clearly define which data are relevant to state each of the diagnoses we obtained, especially those related to [potential] for enhancing. Besides, there is a need for systematic reviews to understand the effectiveness of nursing interventions towards universal self-care requisites needs.

We considered it could be pertinent to compare our findings, based in ICNP $\AA$, to established definitions, diagnoses and statements from other nursing terminologies, such as NANDA, for example. We considered this exciting work to be done afterwards, perhaps even mapping our results with other nursing classifications. It is relevant to involve the National Council (Ordem dos Enfermeiros) in accessing nurses' acceptability of the EHR content we propound. There is already a joint task force between CIDESI and the National Council for developing a nursing ontology to be used in the backend of EHR over time (Ordem dos Enfermeiros 2020).

Our study portrays limitations. We analysed the material provided by the National Ministry of Health, which contains 


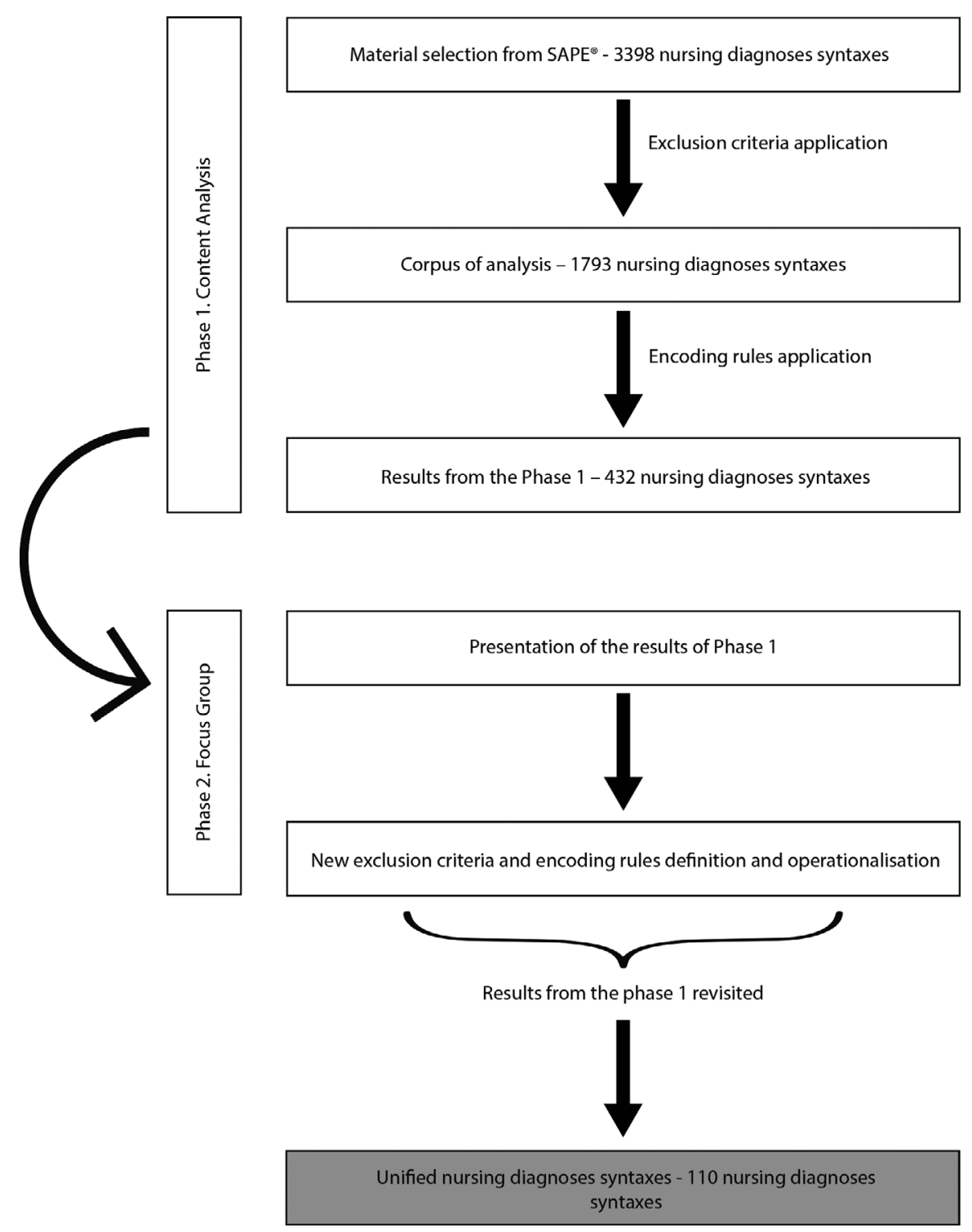

Fig. 1 Summary of findings accordingly with the different phase of the research.

only the SAPE® customizations of public institutions. We also selected the content that incorporates the corpus of analysis based on criteria defined by a research team. This portrait a perspective of viewing the material, which could be different from other nurses in other settings or countries. To manage the enormous amount of retrieved records, we excluded from the corpus all diagnoses related to 'self-care: physical activity' and 'self-care: sleep-rest behaviour'. This could indicate a limitation since these focuses could be considered universal selfcare requisites. The content analysis presented to focus group participants was based on ISO (2003), even though a new standard was released in 2014. According to the new standard, the categorial structure 'dimension' was removed to reduce the combinatorial explosion between concepts. Another limitation of this study was the absence of an external observer in the focus group meeting or videotaping, making it difficult to focus on participants' non-verbal communication and interactions. We only conducted one focus group meeting that allowed data saturation (Goncalves et al. 2019) but did not allow potential differences in the opinion of the participants to be identified over time. Another concern is related to the size of the focus group; if 
we conducted more meetings with smaller groups, the participants could have interacted more, perhaps leading to more in-depth insights. We did not return our findings to the participants, which may restrain additional clarifications.

The results showed that despite Portugal's standout in Europe as a case study for decades of EHR use by nurses, more efforts should be made to produce reliable, generalizable, and feasible data and information.

\section{Conclusions and recommendations}

Nursing diagnoses are a core element of nursing documentation and the basis for selecting effective interventions (Ackley et al. 2019). Our results reveal that nursing diagnoses related to universal self-care requisites can emphasize the individuals' impairment or potential to perform self-care. This study showed a lack of consensus on nominating the nursing diagnoses for individuals with a deficit in universal self-care requisites. If the information that we generate cannot be recognized as efficient and accurate, difficulties in decisionmaking at the patient bedside or governance level will arise between institutions or even within the same institution. This might defy the significant purposes of using EHR with standardized language. Moreover, nursing care and health gains produced by nurses will remain invisible despite all efforts. We support the idea that the solution for this problem relies on research being developed and sustained by evidence and consensus between domain experts that provide an ontology of nursing.

\section{Implications for nursing practice}

Representation of most relevant diagnoses within the scope of universal self-care requisites. The accuracy of the nursing diagnoses determines the implementation of proper nursing interventions, thus better patient outcomes and quality of care.

\section{Implications for nursing policy and health policy}

Our results might provide an essential input towards the standardization of the concepts used by nurses in diagnosis definitions regarding universal self-care requisites. These can lead to health indicators that convey the involvement of nursing care into health. Despite Portugal is at the forefront of eHealth in Europe and stands out as a case study for the use of International Classification for Nursing Practice ${ }^{\circledR}$ into electronic health records, efforts need to be made to enhance edocumentation. It provides exemplary work of what to do and what to avoid in nursing care documentation on to electronic health records. This research sustains the need to integrate a formal representation of nursing knowledge - nursing ontology, into EHR.

\section{Acknowledgement}

The authors wish to acknowledge CIDESI: ICN-Accredited Centre for Information Systems and ICNP® Research and Development, Nursing School of Porto, Porto, Portugal.

\section{Author contributions}

Study design: CQ, MATCPS, IC, AC, EM

Data collection: $\mathrm{CQ}$

Data analysis: CQ, MATCPS

Study supervision: MATCPS

Manuscript writing: CQ

Critical revisions for important intellectual content: CQ, MATCPS, IC, AC, EM

\section{References}

Ackley, B.J., et al. (2019) Nursing Diagnosis Handbook E-Book: An EvidenceBased Guide to Planning Care. Elsevier Health Sciences, St. Louis, MO.

Almeida, M. \& Bax, M. (2003) An overview about ontologies: survey about definitions, types, applications, evaluation and building methods. Ciência da Informação, 32 (3), 7-20.

Bardin, L. (2018) Content analysis. Edições 70, Lisboa (in Portuguese). Brito, A. (2016) Reconstruction of autonomy: an explanatory theory. In The Dependent Person and the Family Caregiver. (Martins, T., Araújo, M.d.F., Peixoto, M.J. \& Machado, P.P. eds), Escola Superior de Enfermagem do Porto (in Portuguese), Porto.

Cardoso, A. \& Sousa, P. (2015) Nursing Informatics Core Competencies for Portugal. HIMSS. Available http://s3.amazonaws.com/rdcms-himss/files/ production/public/FileDownloads/TIGER_HIMSS15_VLE_PortugalCase Study_11182015.pdf. (accessed 16 March 2020).

Carpenito-Moyet, L.J. (2011) Manual of Nursing Diagnoses. Artmed, Porto Alegre (in Portuguese).

Chick, N. \& Meleis, A. (1986) Transitions: a nursing concern. In Nursing Research Methodology. (Chinn, P.L., ed), Aspen Publication, Boulder, CO.

Cruz, I., et al. (2016) Analysis of the Nursing Documentation in Use in Portugal - building a clinical data model of nursing centered on the management of treatment regimen. Studies in Health Technology and Informatics, 225, 407-411.

De Groot, K., Triemstra, M., Paans, W. \& Francke, A.L. (2019) Quality criteria, instruments, and requirements for nursing documentation: A systematic review of systematic reviews. Journal of Advanced Nursing, 75 (7), 1379-1393.

European Observatory on Health Systems and Policies (2018) Health System Review: Portugal, Final Report. World Health Organization.

Friedman, B., Li, Y.E., Liebel, D.V. \& Powers, B.A. (2014) Effects of a home visiting nurse intervention versus care as usual on individual activities of daily living: a secondary analysis of a randomized controlled trial. BMC Geriatrics, 14, 24. (in English).

Gavrilov, G., Vlahu-Gjorgievska, E. \& Trajkovik, V. (2019) Healthcare data warehouse system supporting cross-border interoperability. Health Informatics Journal, 26 (2), 1321-1332. 
Goncalves, P.D.B., Sequeira, C.A.C. \& Paiva e Silva, M.A. (2018) Content analysis of nursing diagnoses in mental health records in Portugal. International Nursing Review, 66 (2), 199-208.

Goncalves, P.D.B., Sequeira, C.A.C. \& Paiva e Silva, M.A. (2019) Nursing interventions in mental health and psychiatry: Content analysis of records from the nursing information systems in use in Portugal. Journal of Psychiatric and Mental Health Nursing, 26 (7-8), 199-211.

Goossen, W. (2000) Towards Strategic Use of Nursing Information in the Netherlands. University of Groningen, Holland.

Hardiker, N.R., Dowding, D., Dykes, P.C. \& Sermeus, W. (2019) Reinterpreting the nursing record for an electronic context. International Journal of Medical Informatics, 127, 120-126.

Harrington, L. (2019) Future model for nursing documentation: extinction. Nurse Leader, 17 (2), 113-116.

International Council of Nurses (2013) International Classification for Nursing Practice. Available https://www.icn.ch/what-we-do/projects/ehea lth/icnp-browser (accessed 6 March 2020) (in Portuguese).

International Council of Nurses (2016) International Classification for Nursing Practice 2015. Ordem dos Enfermeiros, Lisbon (in Portuguese).

International Organization for Standardization (2003) ISO 18104 - Health Informatics: Integration of a Reference Terminology Model for Nursing. International Organization for Standardization, Geneva.

Jones, T.L., Hamilton, P. \& Murry, N. (2015) Unfinished nursing care, missed care, and implicitly rationed care: State of the science review. International Journal of Nursing Studies, 52 (6), 1121-1137.

Kalisch, B.J. (2006) Missed nursing care: a qualitative study. Journal of Nursing Care Quality, 21 (4), 306-3013.

Keenan, G.M. \& Wilkie, D.J. (2014) Integration of NNN into EHRS: How are we doing? International Journal of Nursing Knowledge, 25 (2), 68.

Kitson, A., et al. (2010) Defining the fundamentals of care. International Journal of Nursing Practice, 16 (4), 423-434.

Kitson, A., Robertson-Malt, S. \& Conroy, T. (2013) Identifying the fundamentals of care within Cochrane Systematic reviews: the role of the Cochrane Nursing Care Field Fundamentals of Care Node. International Journal of Nursing Practice, 19 (2), 109-115.

Krueger, R.A. \& Casey, M.A. (2014) Focus Groups: A Practical Guide for Applied Research. Sage publications, Thousand Oaks, CA.

McCarthy, B., et al. (2019) Electronic nursing documentation interventions to promote or improve patient safety and quality care: A systematic review. Journal of Nursing Management, 27 (3), 491-501.

Meleis, A. (2012) Theoretical Nursing: Development \& Progress. Wolters Kluwer| Lippincott Williams \& Wilkins, Philadelphia.

NANDA-International (2015) Diagnósticos de Enfermagem da NANDA: Definições e Classificação 2015-2017. In NANDA International ed. (Herdman, T.H. \& Kamitsuru, S. eds.). Artmed, Porto Alegre (in Portuguese).

Neves, H. \& Parente, P. (2019) A nursing clinical data model for neuromuscular processes: content analysis of the Portuguese nursing customization. Ciência \& Saúde Coletiva, 24 (5), 1609-1616.
Nightingale, F. (1989) Notes on nursing: what is and what is not. Notes on nursing: what is and what is not. (in Portuguese).

Nursing and Midwifery Council. (2018) The Code. Professional Standards of Practice and Behaviour for Nurses, Midwifes and Nursing Associates. Nursing \& Midwifery Council, London.

Ordem dos Enfermeiros. (2007) Nursing Information Systems: Basic Principles of Architecture and Technical-Functional Requirements. Available https://www.ordemenfermeiros.pt/arquivo/documentosoficiais/Docume nts/SIE-PrincipiosBasicosArq_RequisitosTecFunc-Abril2007.pdf. (accessed 16 January 2020) (in Portuguese).

Ordem dos Enfermeiros. (2020) Ordem dos Enfermagem Advances to Improve Nursing Information Systems. Available https://www.ordemenfe rmeiros.pt/noticias/conteudos/ordem-avan\%C3\%A7a-para-a-melhoriados-sistemas-de-informa\%C3\%A7\%C3\%A3o-em-enfermagem/. (accessed 2 March 2020) (in Portuguese).

Orem, D. (1995) Nursing Concepts of Practice. Mosby-Year Book, New York.

Paans, W., et al. (2012) Do knowledge, knowledge sources and reasoning skills affect the accuracy of nursing diagnoses? a randomised study. BMC Nursing, 11 (1), 11.

Paiva, A., et al. (2014) Analysis of the National Parameterization of the Nursing Practice Support System SAPE. Escola Superior de Enfermagem do Porto, Porto (in Portuguese).

Silva, A.P. (2006) Nursing Information Systems: An Explanatory Theory of Change. Formasau, Coimbra (in Portuguese).

Silva, C., Brito, M.A. \& Silva, M.A. (2016) The Person Living with a Stoma: The Formalization of Knowledge in Nursing. $3{ }^{a}$ IPLeiria International Health Congress: Health, Demographic Changes \& Well-being. BMC Health Services Research, Leiria.

Sousa, P.A.F., Dal Sasso, G.T.M. \& Barra, D.C.C. (2012) Contributions of the electronic health records to the safety of intensive care unit patients: an integrative review. Texto \& Contexto Enfermagem, 21 (4), 971-979.

Spigolon, D. \& Moro, C. (2012) Essential data set's archetypes for nursing care of endometriosis patients. Revista Gaúcha de Enfermagem, 33 (4), 22-32. (in Portuguese).

Tastan, S., et al. (2014) Evidence for the existing American Nurses Association-recognized standardized nursing terminologies: A systematic review. International Journal of Nursing Studies, 51 (8), 1160-1170.

World Medical Association (2013) World Medical Association Declaration of Helsinki: ethical principles for medical research involving human subjects. Journal of the American Medical Association, 310 (20), 2191-2194.

World Medical Association (2016) World Medical Association Declaration of Taipei on ethical considerations regarding health databases and biobanks. Available https://www.wma.net/policies-post/wma-declarationof-taipei-on-ethical-considerations-regarding-health-databases-andbiobanks/ accessed 2 June 2020.

Zwakhalen, S.M.G., et al. (2018) Basic nursing care: The most provided, the least evidence based - A discussion paper. Journal of Clinical Nursing, 27 (11-12), 2496-2505. 


\section{Supporting information}

Additional Supporting Information may be found in the online version of this article:

Table S1 Excluded diagnoses from corpus by criteria.

Table S2 Number of diagnoses categories by universal selfcare requisites focus after content analysis.
Table S3 Examples of judgment categories and their degrees (+ICNP codes).

Table S4 Examples of dimensions categories and its specificities (+ICNP codes). 